\title{
Effects of Recombinant Human Bone Morphogenetic Protein-2 on Vertical Bone Augmentation in a Canine Model
}

Yung-Ting Hsu, * Khalid Al-Hezaimi, ${ }^{\dagger}$ Pablo Galindo-Moreno, ${ }^{\ddagger}$ Francisco O’Valle, $§$ Abdulaziz Al-Rasheed, ${ }^{\dagger}$ and Hom-Lay Wang\|

Background: Vertical bone augmentation (VBA) remains unpredictable and challenging for most clinicians. This study aims to compare hard tissue outcomes of VBA, with and without recombinant human bone morphogenetic protein (rhBMP)-2, under space-making titanium mesh in a canine model.

Methods: Eleven male beagle dogs were used in the study. Experimental ridge defects were created to form atrophic ridges. VBA was performed via guided bone regeneration using titanium mesh and allografts. In experimental hemimandibles, rhBMP-2/absorbable collagen sponge was well mixed with allografts prior to procedures, whereas a control buffer was applied within controls. Dogs were euthanized after a 4-month healing period. Clinical and radiographic examinations were performed to assess ridge dimensional changes. In addition, specimens were used for microcomputed tomography (micro-CT) assessment and histologic analysis.

Results: Membrane exposure was found on five of 11 (45.5\%) rhBMP-2-treated sites, whereas it was found on nine of $11(81.8 \%)$ non-rhBMP-2-treated sites. Within 4 months of healing, rhBMP-2-treated sites showed better radiographic bone density, greater defect fill, and significantly more bone gain in ridge height $(P<0.05)$ than controls. Experimental hemimandibles exhibited lower rates of membrane exposure and a noteworthy, ectopic bone formation above the mesh in $72 \%$ of sites. Results from micro-CT also suggested a trend of less vertical bone gain and bone mineral density in controls $(P>0.05)$. Under light microscope, predominant lamellar patterns were found in the specimen obtained from rhBMP-2 sites.

Conclusion: With inherent limitations of the canine model and the concern of such a demanding surgical technique, current findings suggest that the presence of rhBMP-2 in a composite graft allows an increase of vertical gain, with formation of ectopic bone over the titanium mesh in comparison with non-rhBMP-2 sites. J Periodontol 2017;88:896-905.

\section{KEY WORDS}

Alveolar ridge augmentation; biocompatible materials; bone regeneration; dental implants.

\footnotetext{
* Department of Periodontology and Dental Hygiene, University of Detroit Mercy School of Dentistry, Detroit, MI.

$\dagger$ Department of Periodontics and Community Dentistry, 3D Imaging and Biomechanical Laboratory, College of Dentistry, King Saud University, Riyadh, Saudi Arabia.

† Department of Oral Surgery and Implant Dentistry, University of Granada, Granada, Spain.

$\S$ Department of Pathology, School of Medicine and Biopathology and Regenerative Medicine Institute, Centre for Biomedical Research, University of Granada.

|| Department of Periodontics and Oral Medicine, University of Michigan, Ann Arbor, MI.
} 
$\mathrm{R}$ idge deformities, horizontal and/or vertical, lead to both functional and esthetic challenges of dental implant treatments. ${ }^{1}$ A residual ridge generally undergoes significant dimensional changes after tooth extraction. ${ }^{2}$ Therefore, ridge corrective procedures are often recommended to achieve optimal treatment outcomes. Vertical bone augmentation (VBA) has been regarded as one of the greatest challenges due to anatomic limitations and technical difficulties. Numerous surgical modalities that use various materials have been introduced for VBA. ${ }^{3}$ These modalities include, but are not limited to, distraction osteogenesis, ${ }^{4}$ monocortical onlay bone grafting ${ }^{5}$, and guided bone regeneration (GBR). ${ }^{6}$

Based on the concept of compartmentalized wound healing, GBR has been widely used for ridge augmentation via the aids of barrier membranes. ${ }^{7-9}$ Previous researchers advocated predictable and promising outcomes in regaining ridge width. 6,9 In contrast, the amount of vertical bone gain varied from 2 to $8 \mathrm{~mm}$ with a large range of complication ( $0 \%$ to $45.5 \%) .{ }^{3}$ Given undesired wound healing, the majority of complications lead to barrier membrane exposure, which results in loss of graft materials and other unfavorable outcomes, such as infection of bone graft, as well as membrane contamination. ${ }^{10,11}$ For a predictable bone augmentation, principles for GBR were proposed ${ }^{12}$ as key requirements for bone regeneration. These principles, forming the acronym PASS, include the following: 1) primary wound coverage to provide an undisturbed environment for tissue/bone to mature; 2) angiogenesis or blood supply to support needed undifferentiated mesenchymal cells and growth factors in order for the wound to heal; 3) space for tissue/bone to grow; and 4) stability to allow stable wound healing and tissue maturation. ${ }^{12}$

Bone morphogenetic proteins (BMPs) belong to the transforming growth factor $\beta$ superfamily and possess multiple functions. ${ }^{13}$ In addition to neural induction, BMPs act as important signals for bone and cartilage formation, especially in the cell differentiation and matrix synthesis stages. ${ }^{13,14}$ At the cellular level, binding of BMPs to cell membranes brings a cascade of signal transduction to the nucleus, enhancing activation of specific target genes, which are responsible for wound healing and tissue remodeling. ${ }^{15}$ In recent years, several BMPs have been investigated for their osteoinductive potential. ${ }^{16-22}$ In animal models or clinical trials, BMPs were applied in combination with various carrier systems for the purpose of bone regeneration in the procedures of socket grafting, ${ }^{16,19}$ sinus grafting, ${ }^{20,21}$ periodontal repair, ${ }^{22}$ localized bone augmentation, ${ }^{17,23}$ and implant osseointegration. ${ }^{16,24}$ Previous literature has shown that recombinant human bone morphogenetic protein (rhBMP)-2 could not only promote osseointegration, but also induce new bone formation in VBA. ${ }^{25,26}$
Despite the potential benefits of rhBMP-2 on VBA, one main drawback of previous techniques is the use of absorbable membrane as a barrier membrane, since this type of membrane is not rigid enough to hold the space needed for the bone formation because of its inability to prevent overlying soft tissue pressure, especially during VBA. ${ }^{12}$ Titanium meshes (Ti-meshes), therefore, have been applied for GBR because of their outstanding mechanical characteristics, which protect bone materials, prevent mucosal compression, and allow creation of space. ${ }^{27}$ Furthermore, there is only limited evidence available that evaluates the effectiveness of rhBMP-2 under Ti-meshes at both clinical and histologic levels. Hence, the purpose of this study is to compare hard tissue outcomes of VBA, with or without rhBMP-2, under space-making Ti-mesh in a canine model.

\section{MATERIALS AND METHODS}

Eleven 1 -year-old male beagle dogs were used in this study. All procedures involving the dogs were preapproved by the Animal Welfare Committee at King Saud University, Riyadh, Saudi Arabia, and performed at the King Saud University Eng. A.B. Research Center For Growth Factors and Bone Regeneration, Riyadh, Saudi Arabia. In these dogs, vertical defects were created surgically in bimandibular premolar areas after extraction of all premolars. After 4 months of wound healing, vertical GBR was performed using a Ti-mesh 9 and human allografts" in combination with or without rhBMP-2.**

\section{Preparation of Experimental Defects}

All surgical procedures were performed under general anesthesia and localized anesthesia with $2 \%$ xylocaine with 1:200,000 epinephrine. At the beginning of the study (day 0 ), chronic experimental defects were surgically created bilaterally in the mandibles after extraction of all mandibular premolars via a full-thickness flap approach. These subcrestal defects were $30 \times$ $8 \mathrm{~mm}$ (width $\times$ height). Primary closure was carefully achieved to ensure smooth wound healing. The remaining canine dentition was thoroughly cleaned by ultrasonic and hand instrumentation. All of the dogs received regular oral hygiene maintenance and followup at $2,4,8$, and 16 weeks postoperatively until the VBA surgery.

After soft tissue healing, study casts were obtained from residual ridges on bilateral jaws under general anesthesia. A customized measuring stent was later fabricated on each study model using light-cured acrylic resin $^{\dagger \dagger}$ as the reference of ridge alteration. Fitted onto occlusal surfaces of adjacent teeth, this

II Osteo-Mesh, Osteogenics Biomedical, Lubbock, TX.

\# enCore, Osteogenics Biomedical.

* * Infuse, Medtronic, Memphis, TN.

$\dagger \dagger$ Triad TruTray, Dentsply, York, PA. 

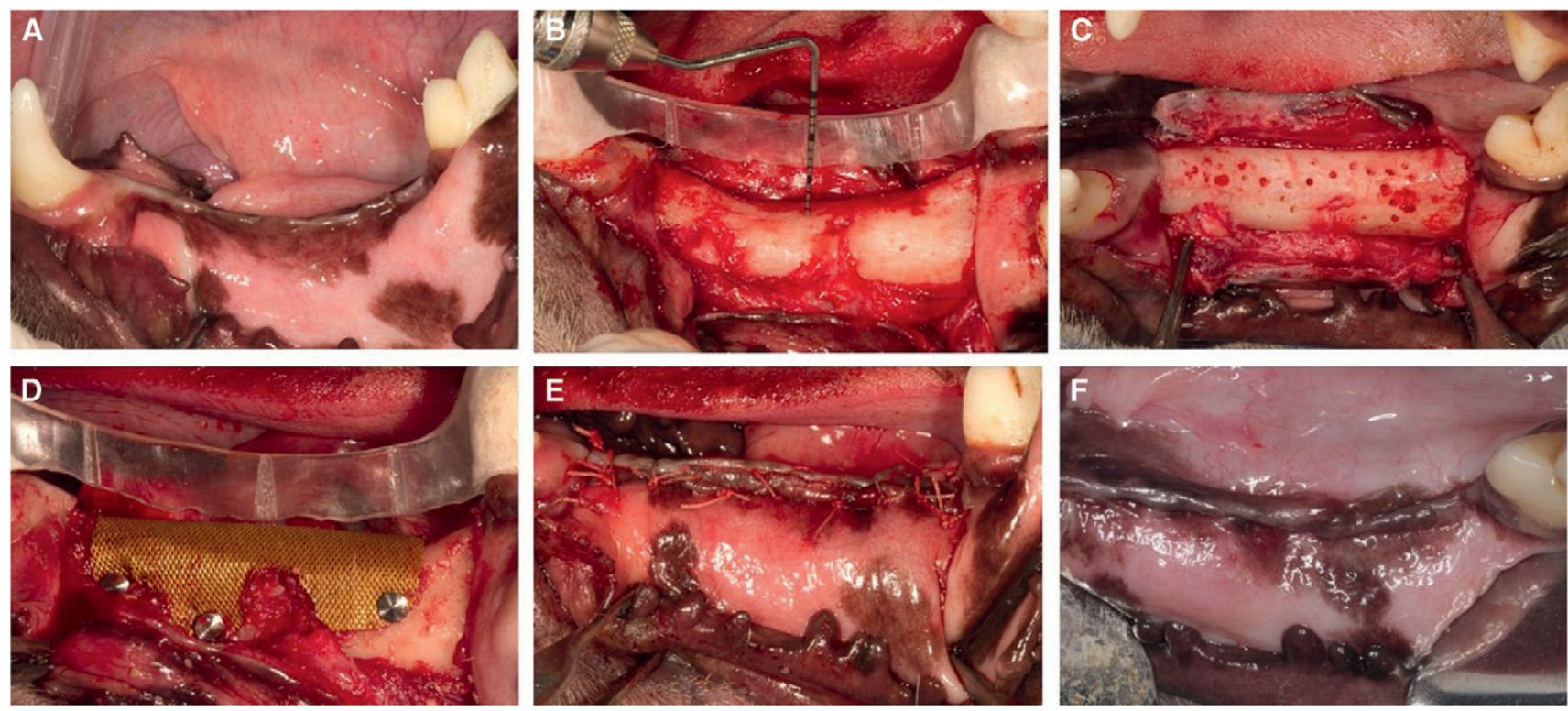

\section{Figure I.}

GBR procedure using allograft and Ti-meshes in combination with rhBMP-2 in the experimental side. A) Chronic ridge defect at 4 months after tooth extractions and ridge creation. B) Preoperative measurement. C) Decortication. D) GBR procedure using allograft and Ti-meshes in combination with rhBMP-2. E) Suture. F) Postoperative view after a healing period of 4 months.

template was grooved in the mesial $(10 \mathrm{~mm}$ away from canine), center (midpoint of the edentulous ridge), and distal aspects ( $10 \mathrm{~mm}$ away from molar) of the residual ridge.

\section{Surgical Procedures}

At day 120 (baseline), VBA was performed using Timeshes and allografts, with (Fig. 1) or without rhBMP-2 (Fig. 2), by calibrated experienced surgeons (HW and PM). A midcrestal incision was made at the chronic experimental defects. Bilateral vertical releasing incisions were performed at the line angles, extending beyond the mucogingival junction. After reflection of full-thickness flaps, the entire ridge defect was exposed and carefully curetted to remove all granulation tissues and soft tissue tags. To promote regional acceleratory phenomenon ${ }^{28}$ and angiogenesis, cortical bone was perforated by a small round bur (\#1/2) until bleeding was noticed. Subsequently, experimental defects were augmented vertically using either the mixture of allografts and rhBMP-2/absorbable collagen sponge (ACS) on the experimental sides (left side), or the combination of allografts and buffer/ACS on the control sides (right sides). Prior to the procedure, an ACS was minced and soaked into either rhBMP-2 $(1.5 \mathrm{mg} / \mathrm{mL})$ or a control buffer for 15 minutes. Carrying rhBMP-2 or control buffer, the minced ACS was well incorporated with allografts and placed on the exposed atrophic ridge until it was level to the adjacent bone. A Ti-mesh was later applied to cover the entire augmented area and stabilized with titanium fixation screws. ${ }^{\ddagger}$ Periosteal releasing incisions were made before suturing to ensure flap approximation and tension-free primary wound closure. Amoxicillin $(25 \mathrm{mg} / \mathrm{kg})$ was administered via intramuscular injection once a day for 5 days to prevent possible infection. Sutures were removed 2 weeks after surgery. Regular plaque control was continued postoperatively, as mentioned previously.

Three dogs were euthanized at day 240, i.e., 4 months after VBA procedures (P4M), using an overdose of 3\% sodium pentobarbital, for histologic analysis and microcomputed tomography (micro-CT) scans. Jaw blocks were obtained from canines to first molar bilaterally.

\section{Clinical and Radiographic Assessments}

Occurrence of adverse events during healing periods were recorded. Clinical and radiographic parameters were assessed to investigate changes of ridge dimension and bone density. Clinically, ridge height alteration (RHA) was defined as vertical distances between the crestal level of soft tissue and the above-mentioned measuring template, taken at baseline (immediately before surgery, RHA1) and P4M (RHA2). Overall vertical bone gain in clinical measurements (VBGc) was calculated as the difference between RHA1 and RHA2. Using the customized measuring template, RHA was measured using a UNC probe $§ \S$ by a single examiner (YH). To assess changes in bone density, standardized periapical radiographs with the aids of bite registration

†† Pro-fix, Osteogenics Biomedical.

$\S \S$ UNC probe, Hu-Friedy, Chicago, IL. 

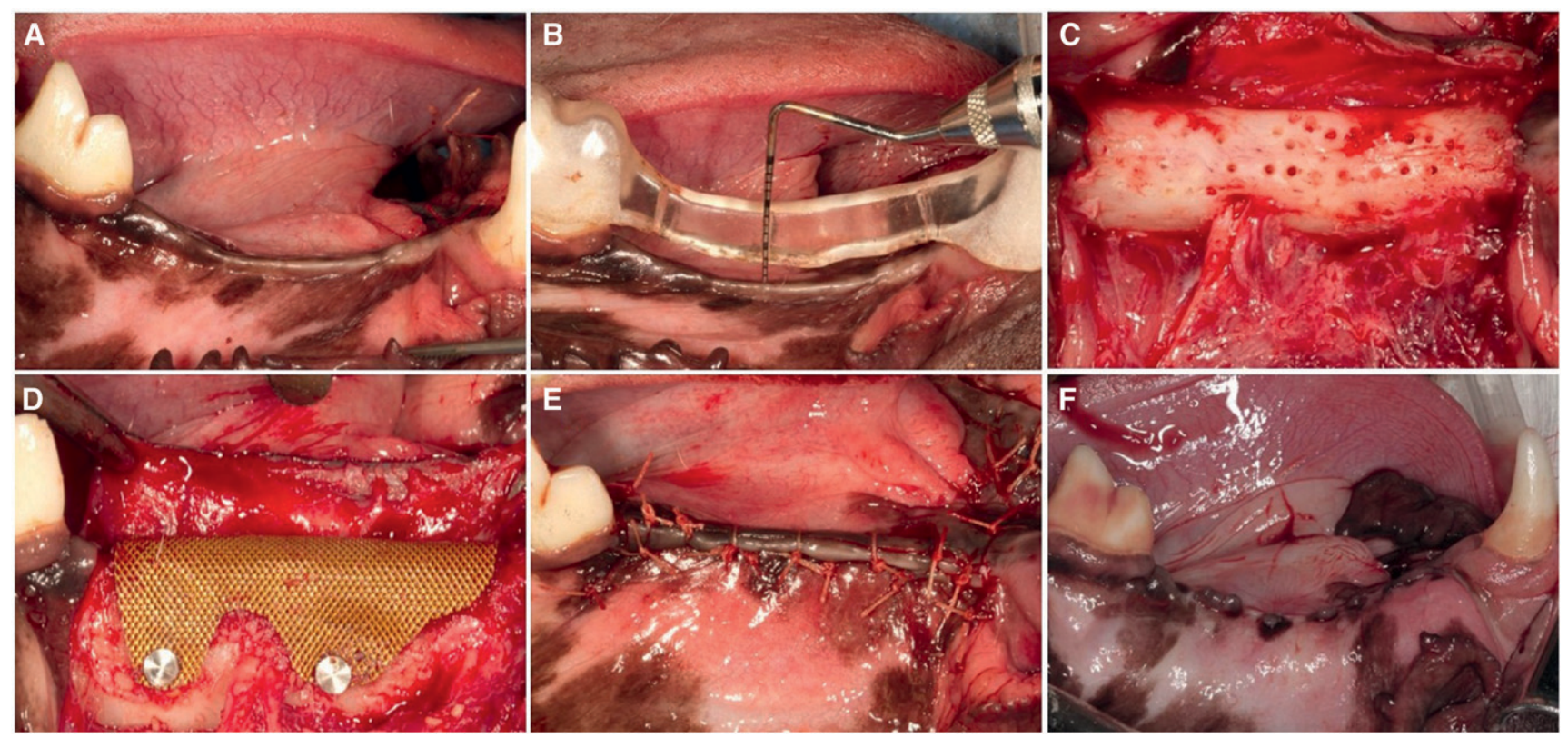

Figure 2.

GBR procedure using allograft and Ti-meshes in the control side. A) Chronic ridge defect at 4 months after tooth extractions and ridge creation.

B) Preoperative measurement. C) Decortication. D) GBR procedure using allograft and Ti-meshes. E) Suture. F) Postoperative view after a healing period of 4 months.

materials were taken immediately before and immediately after GBR and at P4M.

\section{Histologic Analysis and Micro-CT Scans}

As part of the project, only three dogs were euthanized at this point. Therefore, bone mineral density (BMD) and VBG in micro-CT analysis (VBGct) were assessed on these dogs. Jaw blocks obtained from the three dogs were scanned entirely using micro-CT scan assessments prior to specimen preparation. The samples were three-dimensionally evaluated using a micro-CT system IIII with a voltage of $101 \mathrm{kV}$ and current of $96 \mu \mathrm{A}$. Resolution was 37.41- $\mu$ m pixels. Reconstruction and measurements of these data images were performed with evaluation software provided by the manufacturer. Parameters included the following: 1) BMD and 2) VBGct, measuring the increase of vertical ridge height in mesial, center, and distal aspects.

Jaw blocks and tissue samples were immediately fixed in $10 \%$ buffered formalin at room temperature. Because hard tissue formation was observed above and beneath the Ti-meshes on several sites, trephines were used to obtain tissue samples from the regenerated bone, to preserve the layer structure during specimen fixation. The trephines were carefully removed prior to subsequent processes. All samples were decalcified for 20 days and embedded in paraffin. Being divided in an axial plane, $4-\mu \mathrm{m}$ sections were subsequently deparaffinized, rehydrated, and stained with hematoxylin and eosin (H\&E) staining.
Bone histomorphometric analysis was performed semiautomatically on cross sections of augmented hemimandibles using H\&E staining. Ten randomly selected images were assessed using a fluorescence microscope equipped with a $\times 10$ objective lens and a digital microscope camera"t connected to a computer. The images were evaluated by a single examiner (FO) with imaging software ${ }^{\# \# ~}$ to quantify vital bone, which was presented as area and percentages of each component. Using the alveolar crestal levels of first molars as references, percentages of regenerated bone height were also calculated from these specimens.

\section{Statistical Analyses}

Data were analyzed using a statistical analysis software package.*** Results with $P<0.05$ were considered statistically significant. Based upon the power calculation, to reach a power of $0.8,11$ dogs (sample size $=10.17$ ) were required, assuming that the mean difference and standard deviation in bone height gain between the two groups were 2.0 and $1.0 \mathrm{~mm}$, respectively, with $P=0.05$. As a pilot study with limited sample sizes, Wilcoxon signed-rank sum test was used to examine differences of parameters between control and experimental sides and the differences between

I|| SkyScan 1172, CT-Analyser version 1.11.4.2+, SkyScan, Kontich, Belgium.

II DP70, Olympus, Barcelona, Spain

\#\# ImageJ, National Institutes of Health, Bethesda, MD.

*** JMP 6.0. Statistical Discovery, SAS Institute, Cary, NC. 
baseline and P4M. In addition, the Wilcoxon rank sum test was applied to compare differences in results between sites with or without membrane exposure.

\section{RESULTS}

After a 4-month healing period, nine of 11 control sides (81.8\%) experienced membrane exposure, whereas only five of 11 experimental sides (45.5\%) had membrane exposure. In those sites with membrane exposure, moderate inflammation and infection were noticed with the signs of redness, oozing, and notable swelling. All device exposures were noticed $\approx 6$ to 8 weeks after VBA procedures. Membranes were therefore removed once the exposure was noticed. Clinical outcomes are shown in Table 1. No significant differences were found among groups at baseline. From baseline to P4M, RHA barely changed in the control sides in all aspects $(P>0.05)$. In contrast, RHA increased significantly in the experimental sides. In comparisons of experimental and control groups, differences in VBGC reached statistical significance $(P<0.05)$ at the center and distal aspects of the surgical areas. Bone fill was $29.0 \%$ (mesial), $48.4 \%$ (center), and $52.7 \%$ (distal) in the experimental hemimandible, whereas the control groups only had bone fills of $20.4 \%$ (mesial), 8.5\% (center), and -3.1\% (distal).

Table 2 shows results of VBGc measured from sites with or without membrane exposure. In rhBMP-2 groups sites with no membrane exposure had significantly greater $(P<0.05)$ VBGc than those with membrane exposure in mesial and center aspects of surgical fields. Although similar trends were found in controls, differences did not reach statistical significance $(P>0.05)$. At P4M it was also noteworthy that hard tissue was found above the membrane (Fig. 3) in eight of the 11 experimental hemimandibles. However, none of the control sides exhibited the same phenomenon. Obtained by trephine to preserve layer structure, the components of these hard tissues were further investigated by histologic assessment.

Changes of bone density were evaluated using standardized periapical radiographs taken immediately before GBR and at P4M. Bone density was equal in both control and test hemimandibles at baseline. At $\mathrm{P} 4 \mathrm{M}$, bone density on augmented regions increased predominantly in the rhBMP-2-treated hemimandibles, and lower bone density was shown on the controls.

BMD and VBGct (Table 3) were assessed on the three dogs using micro-CT scan analysis. Slightly greater $\mathrm{BMD}$ was also found in the rhBMP-2 groups than controls $\left(1.28 \pm 0.30 \mathrm{~g} / \mathrm{mm}^{3}\right.$ versus $\left.1.13 \pm 0.24 \mathrm{~g} / \mathrm{mm}^{3}\right)$. Nonetheless, differences among groups were not statistically significant $(P>0.05)$. In the control group, VBGct was $4.64 \pm 0.46 \mathrm{~mm}(58.0 \%), 3.49 \pm 0.66 \mathrm{~mm}$ (43.6\%), and $3.59 \pm 0.37 \mathrm{~mm}(44.9 \%)$ at the mesial, center, and distal aspects of the edentulous ridge, respectively. On the other hand, rhBMP-2 hemimandibles gained $6.23 \pm 2.40 \mathrm{~mm}$ (77.9\%), $5.15 \pm 1.40 \mathrm{~mm}$ (64.4\%), and $4.89 \pm 2.48 \mathrm{~mm}(61.3 \%)$ in all three aspects. Although differences between the groups did not reach statistical significance $(P>0.05)$, results showed a trend of greater VBGct in rhBMP-2-treated sites.

Histologic analysis revealed close contacts between new bone and original cortical bone surfaces with no evidence of fibrous tissue intervention. Specimens obtained from experimental sites showed uneventful healing with some signs of chronic inflammation under the light microscope (Fig. 3A). In addition, bone tissue formation was observed above and beneath Ti-meshes (Fig. 3B). Under light microscope observation, hard tissues obtained above Ti-meshes in rhBMP-2 sites were confirmed as newly regenerated bone with a cortical (Figs. 3C and 3D) and trabecular pattern. These specimens showed a predominately lamellar pattern, revealing a Haversian system with concentric lamellae, central canals, and lacunae. In the experimental

\section{Table I.}

\section{Clinical Outcomes: RHA and VBGc (mean \pm SD)}

\begin{tabular}{|c|c|c|c|c|c|c|c|c|}
\hline \multirow{2}{*}{$\begin{array}{l}\text { Group Parameters } \\
\text { Location }\end{array}$} & \multicolumn{4}{|c|}{ Control $(n=||)$} & \multicolumn{4}{|c|}{ Test $(n=1 \mid)$} \\
\hline & $\mathrm{RHAI}(\mathrm{mm})$ & $\mathrm{RHA} 2(\mathrm{~mm})$ & VBG $(\mathrm{mm})$ & Bone fill (\%) & $\mathrm{RHAI}(\mathrm{mm})$ & RHA2 (mm) & VBG $(\mathrm{mm})$ & Bone fill (\%) \\
\hline $\begin{array}{l}\text { Center (midpoint of } \\
\text { the edentulous } \\
\text { ridge) }\end{array}$ & $5.86 \pm 1.98$ & $5.82 \pm 1.78$ & $0.05 \pm 2.20 *$ & 8.53 & $7.23 \pm 1.54^{\dagger}$ & $3.73 \pm 3.69^{\dagger}$ & $3.50 \pm 2.73 *$ & 48.41 \\
\hline $\begin{array}{l}\text { Distal ( } 10 \mathrm{~mm} \text { away } \\
\text { from molar) }\end{array}$ & $5.73 \pm 1.49$ & $5.9| \pm| .5 \mid$ & $-0.18 \pm 1.33 *$ & -3.14 & $6.55 \pm 2.58^{\dagger}$ & $3.09 \pm 2.17^{\dagger}$ & $3.45 \pm 2.67 *$ & 52.67 \\
\hline
\end{tabular}

* Statistically significant $(P<0.05)$ difference in VBG between controls and test sites.

$\dagger$ Statistically significant $(P<0.05)$ difference between RHA1 and RHA2. 
Table 2.

\section{Clinical Outcomes: VBGc (mean \pm SD) in Sites With and Without Membrane Exposure}

\begin{tabular}{lcccccc}
\hline & \multicolumn{4}{c}{ Control } & \multicolumn{3}{c}{ Test } \\
\cline { 2 - 7 } Group & $\begin{array}{c}\text { Membrane } \\
\text { exposure }(\mathrm{mm})\end{array}$ & $\begin{array}{c}\text { Membrane } \\
\text { non-exposure }(\mathrm{mm})\end{array}$ & $P$ value & $\begin{array}{c}\text { Membrane } \\
\text { exposure }(\mathrm{mm})\end{array}$ & $\begin{array}{c}\text { Membrane } \\
\text { non-exposure }(\mathrm{mm})\end{array}$ & $P$ value \\
\hline Number of sites & 9 & 2 & & 5 & 6 & \\
VBGc mesial & $0.36 \pm 2.36$ & $3.00 \pm 1.41$ & 0.23 & $-0.80 \pm 1.10$ & $3.80 \pm 2.38$ & 0.01 * \\
VBGc center & $-0.28 \pm 2.31$ & $1.50 \pm 0.71$ & 0.19 & $1.00 \pm 1.22$ & $5.58 \pm 1.50$ & 0.01 * \\
VBGc distal & $-0.22 \pm 1.39$ & $0 \pm 1.41$ & 0.90 & $2.4 \pm 1.14$ & $4.33 \pm 3.39$ & 0.10 \\
\hline
\end{tabular}

* Statistically significant $(P<0.05)$.

hemimandible, an increase of trabecular bone was also observed morphometrically. Differences among groups reached statistical significance $(P<0.05)$ in favor of rhBMP-2-treated sites $\left(4.06 \pm 2.25 \mathrm{~mm}^{2}\right)$ in comparison with control sites $\left(2.96 \pm 1.62 \mathrm{~mm}^{2}\right)$.

\section{DISCUSSION}

This study examines effects of rhBMP-2 incorporated with human allografts and Ti-meshes for VBA. In the present findings, rhBMP-2 incorporated with human allografts and Ti-meshes appears to achieve greater augmented bone vertically (average of 5.42 versus $3.90 \mathrm{~mm}$ ), greater bone fill and VBGc, and lower risks of membrane exposure than sites treated without rhBMP2. Considering the possible impacts of membrane exposure, results could have been more promising on both sites. No immunologic or adverse reactions were noticed in the histologic level from the present results. Under light microscopic observation, samples from rhBMP-2 groups exhibited more mineral components of newly formed tissues. It appeared in the present results that rhBMP-2 possesses regenerative potential in spite of potential adverse effects of rhBMP-2.

In the present study, ridge defects were created and re-entered after a healing period of 4 months. These chronic ridge defects were made purposely to reduce potential for regeneration, which is commonly observed in a freshly created defect. Results showed that rhBMP-2 may benefit vertical bone gain with the application of GBR. Mean bone fills were $29.0 \%, 48.4 \%$, and $52.7 \%$ in mesial, center, and distal aspects of experimental hemimandibles, respectively. Meanwhile, controls exhibited minimal gain or even loss of vertical bone during the study period. These results corresponded well with previous findings from other animal models. ${ }^{15,17,25}$ In a series of studies, Wikesjö and coworkers ${ }^{15,25}$ examined the regenerative potential of rhBMP-2 along with a calcium-phosphate cement carrier, using a model with a $5 \mathrm{~mm}$ suprabony defect created surgically around implants. Promising results were observed in
rhBMP-2-treated groups, including radiographic bone regeneration and clinically uneventful healing. In histometric analysis, $89 \%$ to $95 \%$ of defect height was made up of regenerated bone in rhBMP-2-treated sites compared with $7 \%$ in controls. ${ }^{15}$ Subsequently, the potential of rhBMP-2 in VBA was further investigated in GBR using expanded polytetrafluoroethylene (ePTFE) membrane for space creation. Compared with controls, an additional 2.9- to 3.5-mm vertical bone gain was obtained in sites treated with rhBMP-2/ACS. ${ }^{25}$ Investigating results from GBR procedures, the same group reported higher bone fill in sites receiving rhBMP-2/ACS or rhBMP-2/GBR than controls. ${ }^{17}$ Indeed, a more recent study proved additional benefits of the bone substitute materials used in conjunction with rhBMP-2/ACS in an animal GBR model. ${ }^{26}$ From these studies, it can be suggested that addition of bone graft materials to the rhBMP-2 enhances predictability of VBA.

From a tissue-engineering standpoint, use of Timesh not only provides space needed for rhBMP-2/ ACS to induce bone formation but also directs the geometry of newly formed bone. ${ }^{29}$ Adopting these principles, de Freitas and coworkers ${ }^{30}$ achieved promising horizontal ridge augmentation from rhBMP-2/ ACS application in combination with Ti-meshes, which provided space creation and wound stability. In the present study, it is noteworthy that $72.7 \%$ of experimental sites exhibited ectopic bone formation to a certain degree, which was rarely reported previously. This phenomenon could be ascribed to the rapid and strong interaction between proteins and scaffolds. ${ }^{31,32}$ Bone regeneration using direct gene delivery of BMPs has been investigated in ectopic animal models, suggesting the osteogenesis properties of these proteins. ${ }^{31}$ Depending on the size of the pores and interconnectivity of protein and grafting materials, previous researchers claimed penetration of bone tissue results from rapid protein resorption to the porous alloplastic materials used in their models and is therefore limited to 

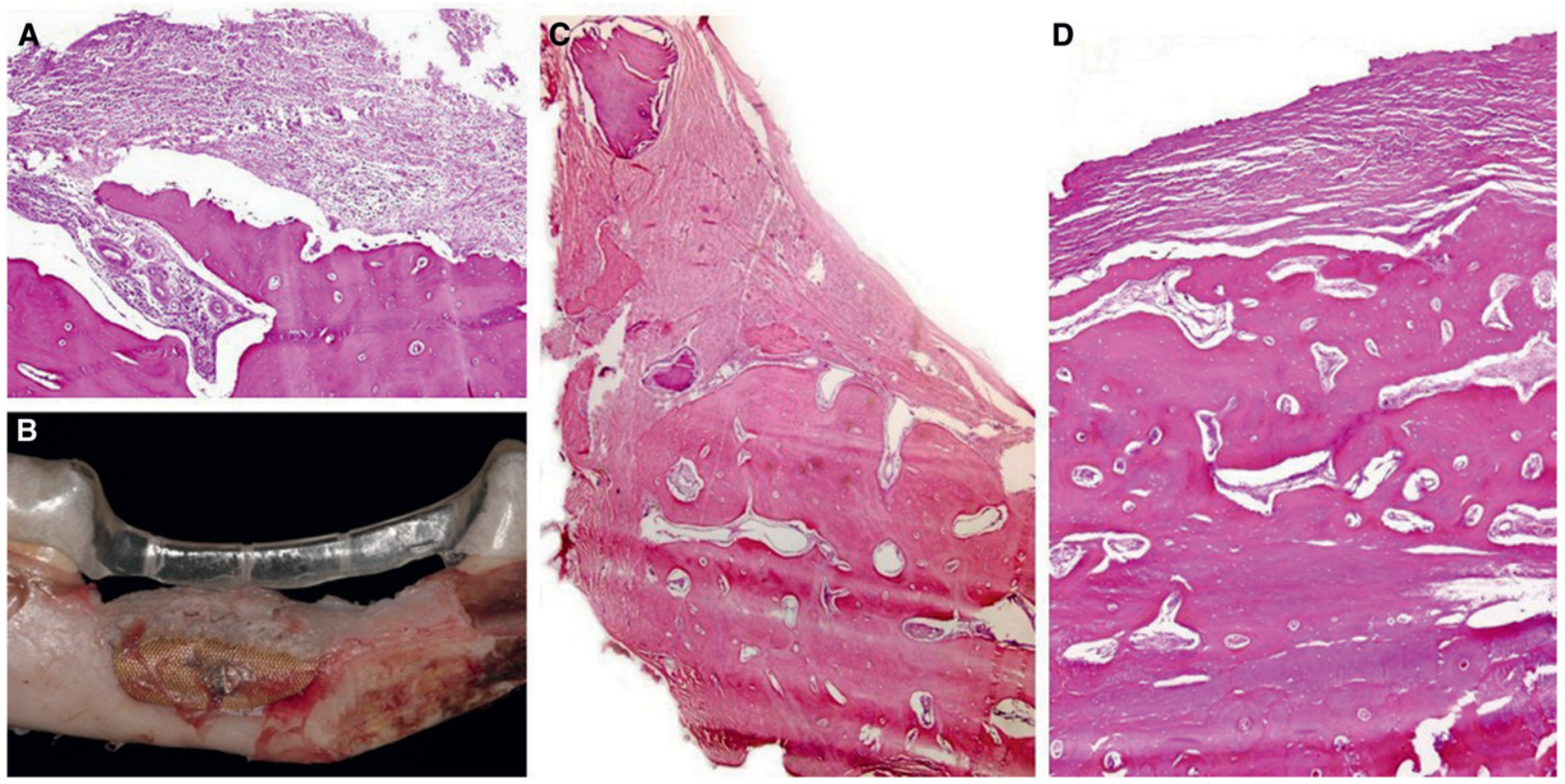

Figure 3.

Hard tissue outcomes in the experimental side. A) Photomicrograph of mandible bone tissue in area of experimental defect. Note the severe inflammatory infiltrate on the surface. (H\&E; original magnification $\times 4$.) B) Photograph of mandible section of dog on the experimental side. New bone formation above Ti-mesh in a test animal treated with allograft plus rhBMP-2 can be observed. C) Photomicrograph of vascularized cancellous bone formed above titanium mesh with an area of mesenchymal tissue showing the remaining bone allograft tissue. (H\&E; original magnification $\times 4$.) D) Photomicrograph of mandible bone tissue in experimental defect area. Note connective tissue and absence of inflammatory infiltrate over new cancellous bone tissue in an animal treated with allograft plus rhBMP-2 (test group). (H\&E; original magnification $\times 4$.)

the periphery of scaffolds. ${ }^{32}$ From the anatomic shape of the new vital bone over the Ti-mesh in the current study, the authors suggest the ectopic bone formation may be related to mesenchymal cells from the periosteum instead of from underlying bone.

Success of GBR relies on primary closure of the soft tissues. In fact, membrane exposure has been considered a critical drawback of GBR. ${ }^{3}$ Although Lindfors et al. ${ }^{33}$ suggested that membrane exposure may not show a negative impact on GBR outcomes using a titanium-reinforced ePTFE membrane, both metaanalysis and clinical studies suggested that exposure of barrier devices often impair GBR outcomes, ${ }^{34,35}$ resulting from inflammatory tissue reactions and bacterial contamination. ${ }^{36,37}$ A previous human study using titanium-reinforced barriers on VBA showed

\section{Table 3.}

Micro-CT Assessment: VBGct and BMD

\begin{tabular}{|c|c|c|c|c|c|}
\hline Dog Number & Location & \multicolumn{3}{|c|}{ VBGct (mm) } & $\mathrm{BMD}\left(\mathrm{g} / \mathrm{mm}^{3}\right)$ \\
\hline I & Test (left) & 4.39 & 5.05 & 4.84 & 0.94 \\
\hline 2 & Control (right) & 4.84 & 4.25 & 3.80 & 1.23 \\
\hline 3 & Test (left) & 8.95 & 6.60 & 7.40 & 1.52 \\
\hline \multirow[t]{2}{*}{ Mean \pm SD (\%) } & Control (right) & $4.64 \pm 0.46(58.0)$ & $3.49 \pm 0.66(43.63)$ & $3.59 \pm 0.37(44.88)$ & $1.13 \pm 0.24$ \\
\hline & Test (left) & $6.23 \pm 2.40(77.9)$ & $5.15 \pm 1.40(64.4)$ & $4.89 \pm 2.48(61.3)$ & $1.28 \pm 0.30$ \\
\hline
\end{tabular}


a complication rate of $40 \% .{ }^{38}$ In addition, $42 \%$ of membrane exposure rate (three of seven of the sites) was reported in an earlier canine study using rhBMP-2 for bone reconstruction. ${ }^{17}$ These results corresponded to the current findings. Since most of the complications occurred in the first group of dogs, the authors believe the occurrence of membrane exposure could be ascribed to the learning curve during surgical procedures. The high membrane exposure rate in the current study could be ascribed to the learning curve of the research team in terms of preparation protocol, surgical procedures, and postoperative care protocol. In addition to postoperative tissue retraction due to flap edema and inflammatory responses, animal behavior regarding their exertion of detrimental biting mechanism for pain relief could be another explanation. It is presumed that any effect of these animal-mediated conditions could occur in one animal with the same intensity in the experimental as in the control site. Despite the surgeries being conducted by the same surgeons, with the same materials (except the application of rhBMP-2), and the splitmouth design, a lower percentage of membrane exposure $(45.5 \%)$ was found on the test sites than the controls $(81.8 \%)$ in the present study. In previous literature, endogenous BMPs have been confirmed to activate the BMP pathway in blood vessels and enhance tissue repair through angiogenesis by inducing vascular endothelial growth factor-A or through pericyte activation. ${ }^{39}$ Given reports in the literature and the present results, it appears that experimental sites healed more quickly than control sites. One could speculate that exogenous BMP may have a role in accelerating angiogenesis in the wound in the very early stages, rather than this being a miraculous effect of the protein.

Usage of BMPs shows some controversial aspects as differentiation factors or as chemotactic agents. ${ }^{14}$ They stimulate angiogenesis, migration, proliferation, and differentiation of stem cells from the surrounding mesenchymal tissues in an area of injury. ${ }^{14}$ As a genetically engineered human protein, rhBMP-2 is secreted from Chinese hamster ovary cells encoding the human rhBMP-2 protein gene and is approved for spinal fusion and tibia repair by the US Food and Drug Administration (FDA). Certain oral and maxillofacial uses, such as sinus augmentation and localized alveolar ridge augmentation used for the defect associated with the extraction socket, were also approved by the FDA. ${ }^{40}$ However, the controversy surrounding agents has been raised recently in regard to multiple complications associated with spinal surgeries. As a reflection, the Yale University Open Data Access (YODA) Project was conducted to review these concerns. ${ }^{41}$ In 2013, two reports ${ }^{42,43}$ were published with several important findings: 1) there was no significant difference in fusion rates between rhBMP- 2 and iliac crest autograft; 2) similar rates of retrograde ejaculation and neurologic complications were found to be associated with the uses of rhBMP-2 and autograft iliac crest bone graft in the procedures of anterior cervical fusion or posteriolateral fusion; 3) high complication rates were found in anterior cervical fusion and posterior lumbar procedures in terms of ectopic bone formation; and 4) the absolute risk of cancer with use of BMP-2 remained minimal. ${ }^{42}$ Similar concerns of carcinogenesis have been raised with regard to use of BMP-2 in oral and maxillofacial procedures. ${ }^{44,45}$ In vitro and animal studies showed an adverse effect on cell invasion and potential effects on oral squamous cell carcinoma, thereby raising concerns for the safety of rhBMP-2 applications for bone reconstruction in patients with oral cancer. ${ }^{43,44}$ In spite of these concerns, the authors recommend considering the combination of BMP-2 and GBR as a possible alternative for bone reconstruction in selected healthy patients when the iliac crest autograft is not available because of the following reasons: 1) similar regenerative potential of bone formation can be achieved in both approaches: combination of BMP-2 and GBR had fewer expenses and less morbidity compared with iliac autografts; ${ }^{42,43,46}$ 2) the absolute risk of cancer with the use of BMP-2 remained minimal as results of YODA projects show; 3 ) to date, no clinical report in the dental field has shown the presence of life-threatening complications, such as swallowing/breathing difficulties, after use of BMP-2 in sinus lifts, socket augmentation, or ridge augmentation. Nevertheless, it is the responsibility of the clinician to inform the patient about these risks prior to treatment.

Limitations of this study include the small sample sizes, short experimental periods, and the lower evidence level associated with an animal model compared with clinical trials. To facilitate the investigation of healing outcomes, qualifying measurements of initial soft tissue healing and quantities of soft tissue flap should be included to evaluate contributing factors of membrane exposure. Clinical trials with longer follow-up time are still needed to provide the impacts of rhBMP-2 on VBA in a long-term setting.

\section{CONCLUSIONS}

With inherent limitations of the canine model and the concern of such a demanding surgical technique, current findings suggest that presence of rhBMP-2 in combination with allograft allows an increase of vertical gain with formation of ectopic bone over the Ti-mesh compared with non-rhBMP-2 sites. Based on histologic analysis, rhBMP-2 may induce vital bone formation within a healing period of 4 months. Further studies are necessary with a larger population to evaluate the longterm effects of rhBMP- 2 on bone regeneration in humans, 
as well as the potential adverse effects of rhBMP- 2 on clinical oral and maxillofacial uses.

\section{ACKNOWLEDGMENTS}

This paper was partially supported by the University of Michigan Periodontal Graduate Student Research Fund (Ann Arbor, MI) and the King Saud University Eng. A.B. Research Center for Growth Factors and Bone Regeneration (Riyadh, Saudi Arabia). In addition, the BMP-2 used in the study was kindly donated by Dentium (Seoul, South Korea). The authors also thank Dr. Antony Neely (Associate Professor, Department of Periodontology and Dental Hygiene, University of Detroit Mercy School of Dentistry, Detroit, MI) and Ms. Eleanor Oster (candidate at the University of Detroit Mercy, Detroit, MI) for advice in manuscript preparation and language editing. The authors report no conflicts of interest related to this study.

\section{REFERENCES}

1. Wang HL, Al-Shammari K. HVC ridge deficiency classification: A therapeutically oriented classification. Int $J$ Periodontics Restorative Dent 2002;22:335-343.

2. Schropp L, Wenzel A, Kostopoulos L, Karring T. Bone healing and soft tissue contour changes following singletooth extraction: A clinical and radiographic 12-month prospective study. Int $J$ Periodontics Restorative Dent 2003;23:313-323.

3. Rocchietta I, Fontana F, Simion M. Clinical outcomes of vertical bone augmentation to enable dental implant placement: A systematic review. J Clin Periodontol 2008;35(Suppl. 8):203-215.

4. Chiapasco M, Consolo U, Bianchi A, Ronchi P. Alveolar distraction osteogenesis for the correction of vertically deficient edentulous ridges: A multicenter prospective study on humans. Int J Oral Maxillofac Implants 2004; 19:399-407.

5. Nyström E, Ahlqvist J, Kahnberg KE, Rosenquist JB. Autogenous onlay bone grafts fixed with screw implants for the treatment of severely resorbed maxillae. Radiographic evaluation of preoperative bone dimensions, postoperative bone loss, and changes in soft-tissue profile. Int J Oral Maxillofac Surg 1996;25:351-359.

6. Simion M, Trisi P, Piattelli A. Vertical ridge augmentation using a membrane technique associated with osseointegrated implants. Int J Periodontics Restorative Dent 1994; 14:496-511.

7. Simion M, Dahlin C, Rocchietta I, Stavropoulos A, Sanchez R, Karring T. Vertical ridge augmentation with guided bone regeneration in association with dental implants: An experimental study in dogs. Clin Oral Implants Res 2007;18:86-94.

8. Buser D, Dula K, Hirt HP, Schenk RK. Lateral ridge augmentation using autografts and barrier membranes: A clinical study with 40 partially edentulous patients. Int J Oral Maxillofac Surg 1996;54:420-432.

9. Urban IA, Jovanovic SA, Lozada JL. Vertical ridge augmentation using guided bone regeneration (GBR) in three clinical scenarios prior to implant placement: A retrospective study of 35 patients 12 to 72 months after loading. Int J Oral Maxillofac Implants 2009;24:502-510.

10. Mau LP, Cheng CW, Hsieh PY, Jones AA. Biological complication in guided bone regeneration with a poly- lactic acid membrane: A case report. Implant Dent 2012;21:171-174.

11. Gher ME, Quintero G, Assad D, Monaco E, Richardson AC. Bone grafting and guided bone regeneration for immediate dental implants in humans. J Periodontol 1994;65:881-891.

12. Wang HL, Boyapati L. "PASS" principles for predictable bone regeneration. Implant Dent 2006;15:8-17.

13. Chen ST, Wilson TG Jr., Hämmerle CH. Immediate or early placement of implants following tooth extraction: Review of biologic basis, clinical procedures, and outcomes. Int J Oral Maxillofac Implants 2004;(Suppl. 19): 12-25.

14. Huang YH, Polimeni G, Qahash M, Wikesjö UM. Bone morphogenetic proteins and osseointegration: Current knowledge - Future possibilities. Periodontol 20002008 ; 47:206-223.

15. Wikesjö UM, Sorensen RG, Kinoshita A, Wozney JM. rhBMP-2/alphaBSM induces significant vertical alveolar ridge augmentation and dental implant osseointegration. Clin Implant Dent Relat Res 2002;4:174-182.

16. Cochran DL, Jones AA, Lilly LC, Fiorellini JP, Howell H. Evaluation of recombinant human bone morphogenetic protein-2 in oral applications including the use of endosseous implants: 3-year results of a pilot study in humans. J Periodontol 2000;71:1241-1257.

17. Jovanovic SA, Hunt DR, Bernard GW, Spiekermann H, Wozney JM, Wikesjö UM. Bone reconstruction following implantation of rhBMP-2 and guided bone regeneration in canine alveolar ridge defects. Clin Oral Implants Res 2007;18:224-230.

18. Thoma DS, Jones A, Yamashita M, Edmunds R, Nevins $M$, Cochran DL. Ridge augmentation using recombinant bone morphogenetic protein-2 techniques: An experimental study in the canine. J Periodontol 2010; 81:1829-1838.

19. Fiorellini JP, Howell TH, Cochran D, et al. Randomized study evaluating recombinant human bone morphogenetic protein-2 for extraction socket augmentation. J Periodontol 2005;76:605-613.

20. Nevins M, Kirker-Head C, Nevins M, Wozney JA, Palmer R, Graham D. Bone formation in the goat maxillary sinus induced by absorbable collagen sponge implants impregnated with recombinant human bone morphogenetic protein-2. Int J Periodontics Restorative Dent 1996;16:8-19.

21. Triplett RG, Nevins M, Marx RE, et al. Pivotal, randomized, parallel evaluation of recombinant human bone morphogenetic protein-2/absorbable collagen sponge and autogenous bone graft for maxillary sinus floor augmentation. J Oral Maxillofac Surg 2009;67:1947-1960.

22. King GN, Cochran DL. Factors that modulate the effects of bone morphogenetic protein-induced periodontal regeneration: A critical review. J Periodontol 2002;73:925-936.

23. Jung RE, Windisch SI, Eggenschwiler AM, Thoma DS, Weber FE, Hämmerle CH. A randomized-controlled clinical trial evaluating clinical and radiological outcomes after 3 and 5 years of dental implants placed in bone regenerated by means of GBR techniques with or without the addition of BMP-2. Clin Oral Implants Res 2009;20:660-666.

24. Gutwald R, Haberstroh J, Stricker A, et al. Influence of rhBMP-2 on bone formation and osseointegration in different implant systems after sinus-floor elevation. An in vivo study on sheep. J Craniomaxillofac Surg 2010; 38:571-579. 
25. Wikesjö UM, Qahash M, Thomson RC, et al. rhBMP-2 significantly enhances guided bone regeneration. Clin Oral Implants Res 2004;15:194-204.

26. Polo CI, Lima JL, De Lucca L, et al. Effect of recombinant human bone morphogenetic protein 2 associated with a variety of bone substitutes on vertical guided bone regeneration in rabbit calvarium. $J$ Periodontol 2013;84:360-370.

27. Rakhmatia YD, Ayukawa Y, Furuhashi A, Koyano K. Current barrier membranes: Titanium mesh and other membranes for guided bone regeneration in dental applications. J Prosthodont Res 2013;57:3-14.

28. Frost HM. The regional acceleratory phenomenon: A review. Henry Ford Hosp Med J 1983;31:3-9.

29. Wikesjö UM, Qahash M, Thomson RC, et al. Spaceproviding expanded polytetrafluoroethylene devices define alveolar augmentation at dental implants induced by recombinant human bone morphogenetic protein 2 in an absorbable collagen sponge carrier. Clin Implant Dent Relat Res 2003;5:112-123.

30. de Freitas RM, Susin C, Spin-Neto R, et al. Horizontal ridge augmentation of the atrophic anterior maxilla using rhBMP-2/ACS or autogenous bone grafts: A proof-of-concept randomized clinical trial. J Clin Periodontol 2013;40:968-975.

31. Rose LC, Kucharski C, Uludağ H. Protein expression following non-viral delivery of plasmid DNA coding for basic FGF and BMP-2 in a rat ectopic model. Biomaterials 2012;33:3363-3374.

32. van de Watering FC, van den Beucken JJ, van der Woning SP, et al. Non-glycosylated BMP-2 can induce ectopic bone formation at lower concentrations compared to glycosylated BMP-2. J Control Release 2012; 159:69-77.

33. Lindfors LT, Tervonen EA, Sándor GK, Ylikontiola LP. Guided bone regeneration using a titanium-reinforced ePTFE membrane and particulate autogenous bone: The effect of smoking and membrane exposure. Oral Surg Oral Med Oral Pathol Oral Radiol Endod 2010; 109:825-830.

34. Machtei EE. The effect of membrane exposure on the outcome of regenerative procedures in humans: A meta-analysis. J Periodontol 2001;72:512-516.

35. Nowzari H, Slots J. Microbiologic and clinical study of polytetrafluoroethylene membranes for guided bone regeneration around implants. Int $J$ Oral Maxillofac Implants 1995;10:67-73.

36. Selvig KA, Kersten BG, Chamberlain AD, Wikesjö UM, Nilvéus RE. Regenerative surgery of intrabony periodontal defects using ePTFE barrier membranes: Scanning electron microscopic evaluation of retrieved membranes versus clinical healing. JPeriodontol 1992; 63:974-978.

37. De Sanctis M, Zucchelli G, Clauser C. Bacterial colonization of bioabsorbable barrier material and periodontal regeneration. J Periodontol 1996;67:1193-1200.

38. Merli M, Migani M, Esposito M. Vertical ridge augmentation with autogenous bone grafts: Resorbable barriers supported by ostheosynthesis plates versus titaniumreinforced barriers. A preliminary report of a blinded, randomized controlled clinical trial. Int $J$ Oral Maxillofac Implants 2007;22:373-382.

39. Yu YY, Lieu S, Lu C, Miclau T, Marcucio RS, Colnot C. Immunolocalization of BMPs, BMP antagonists, receptors, and effectors during fracture repair. Bone 2010; 46:841-851.

40. United States Food and Drug Administration. PMA P050053: FDA summary of safety and effectiveness data. Available at: http://www.accessdata.fda.gov/cdrh_ docs/pdf5/P050053b.pdf. Accessed March 29, 2017.

41. Hustedt JW, Blizzard DJ. The controversy surrounding bone morphogenetic proteins in the spine: A review of current research. Yale J Biol Med 2014;87:549-561.

42. Fu R, Selph S, McDonagh M, et al. Effectiveness and harms of recombinant human bone morphogenetic protein-2 in spine fusion: A systematic review and meta-analysis. Ann Intern Med 2013;158:890-902.

43. Simmonds MC, Brown JV, Heirs MK, et al. Safety and effectiveness of recombinant human bone morphogenetic protein-2 for spinal fusion: A meta-analysis of individualparticipant data. Ann Intern Med 2013;158:877-889.

44. Kokorina NA, Lewis JS Jr., Zakharkin SO, Krebsbach $\mathrm{PH}$, Nussenbaum B. rhBMP-2 has adverse effects on human oral carcinoma cell lines in vivo. Laryngoscope 2012;122:95-102.

45. Kim MJ, Kim KM, Kim J, Kim KN. BMP-2 promotes oral squamous carcinoma cell invasion by inducing CCL5 release. PLoS One 2014;9:e108170.

46. Dahlin C, Johansson A. Iliac crest autogenous bone graft versus alloplastic graft and guided bone regeneration in the reconstruction of atrophic maxillae: A 5-year retrospective study on cost-effectiveness and clinical outcome. Clin Implant Dent Relat Res 2011;13:305-310.

Correspondence: Dr. Yung-Ting Hsu, Department of Periodontology and Dental Hygiene, University of Detroit Mercy School of Dentistry, 2700 Martin Luther King Jr. Blvd., Detroit, MI 48208-2576. Fax: 313/494-6666; e-mail: hsuyu2@udmercy.edu.

Submitted August 4, 2016; accepted for publication April 5, 2017. 